\title{
A neutron source for IGISOL-JYFLTRAP: Design and characterisation
}

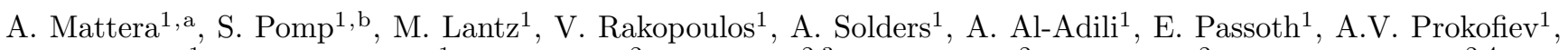 \\ P. Andersson ${ }^{1}$, A. Hjalmarsson ${ }^{1}$, R. Bedogni ${ }^{2}$, D. Bortot ${ }^{2,3}$, A. Esposito ${ }^{2}$, A. Gentile ${ }^{2}$, J.M. Gómez-Ros ${ }^{2,4}$, \\ M.V. Introini ${ }^{3}$, A. Pola ${ }^{3}$, D. Gorelov ${ }^{5}$, H. Penttilä ${ }^{5}$, I.D. Moore ${ }^{5}$, S. Rinta-Antila ${ }^{5}$, V.S. Kolhinen ${ }^{5}$, and T. Eronen ${ }^{5}$ \\ 1 Uppsala University, BOX 516, 75120 Uppsala, Sweden \\ 2 INFN-LNF, via E. Fermi 40, 00044 Frascati (RM), Italy \\ 3 Politecnico di Milano, p.zza L. da Vinci 32, 20133 Milano, Italy \\ 4 CIEMAT, Complutense 40, 28040 Madrid, Spain \\ 5 University of Jyväskylä, P.O. Box 35 (YFL), 40014 Jyväskylä, Finland
}

Received: 14 May 2017 / Revised: 20 July 2017

Published online: 30 August 2017

(C) The Author(s) 2017. This article is published with open access at Springerlink.com

Communicated by A. Jokinen

\begin{abstract}
A white neutron source based on the $\mathrm{Be}(\mathrm{p}, \mathrm{nx})$ reaction for fission studies at the IGISOLJYFLTRAP facility has been designed and tested. $30 \mathrm{MeV}$ protons impinge on a $5 \mathrm{~mm}$ thick water-cooled beryllium disc. The source was designed to produce at least $10^{12}$ fast neutrons/s on a secondary fission target, in order to reach competitive production rates of fission products far from the valley of stability. The Monte Carlo codes MCNPX and FLUKA were used in the design phase to simulate the neutron energy spectra. Two experiments to characterise the neutron field were performed: the first was carried out at The Svedberg Laboratory in Uppsala (SE), using an Extended-Range Bonner Sphere Spectrometer and a liquid scintillator which used the time-of-flight (TOF) method to determine the energy of the neutrons; the second employed Thin-Film Breakdown Counters for the measurement of the TOF, and activation foils, at the IGISOL facility in Jyväskylä (FI). Design considerations and the results of the two characterisation measurements are presented, providing benchmarks for the simulations.
\end{abstract}

\section{Introduction}

Accurate measurements of neutron-induced fission yields (FY) are desirable in order to obtain a better understanding of the fission process. Of particular importance are isotopic yields of short-lived, very neutron-rich nuclei far from the line of stability, for which data are very scarce. Measurements with techniques of high mass resolving power also allow, e.g., determination of isomeric fission yields, which are relevant for the extraction of the spin of the initial fragments.

Even for the most well-studied nuclides and primary beam energies, such as the fission of ${ }^{235} \mathrm{U}$ induced by thermal neutrons, the uncertainties in evaluated nuclear data libraries for the lower-yield isotopes can be as high as $50 \%$ [1]. For fission induced by higher-energy neutrons, the landscape of available measurements is even less populated and the uncertainties of the available experimental and evaluated data are larger. The conclusions from a re-

\footnotetext{
a e-mail: andrea.mattera@physics.uu.se

b e-mail: stephan.pomp@physics.uu.se
}

cent workshop hosted by the IAEA [2] highlight the need for new high-quality measurements of FY both for fundamental research (e.g., development of models in the field of nuclear astrophysics), and to improve the knowledge of the fission product content of (spent) nuclear fuel.

The IGISOL-JYFLTRAP facility at the University of Jyväskylä (JYFL) has been used in the past years for measurements of proton-induced fission yields $[3,4]$ and studies of nuclei far from the valley of stability produced in fission reactions. The installation of a proton-neutron converter will offer access to the production of even more neutron-rich nuclei for nuclear structure studies [5-7], but also to measurements of neutron-induced FY. The wide energy spread of the neutrons produced by the source described in this work will offer the possibility to perform integral measurements of $\mathrm{FY}$ over the $0-30 \mathrm{MeV}$ range; measurements in this energy range are very scarce and new data - even with a wide input spectrum - provide a valuable contribution in benchmarking fission models and nuclear data evaluations. In the forthcoming experimental campaigns, we will be able to study FY in different energy intervals by varying the incoming proton energy 
and/or the target thickness; or by including a moderator inside the fission chamber to obtain a thermal spectrum.

In order to ensure a sufficiently short measurement time even for fission products with lower yields, the aim is to obtain a fission rate comparable to the earlier protoninduced experiments. This can be achieved working concurrently on the neutron converter design, impinging proton current and amount of fissionable material. Since the production of nuclei far from the line of stability is an important objective of the facility, a lower limit on the intensity of neutrons with energies above $1 \mathrm{MeV}$ was set to $10^{12} \mathrm{n} / \mathrm{s}$ as a design goal [8].

With the latest upgrade to IGISOL-4 [9], the facility has been equipped with a high-current MCC30/15 cyclotron that can deliver protons up to $30 \mathrm{MeV}$ at $100 \mu \mathrm{A}$ and deuterons of half that energy and current. Despite the fact that the neutron yield per incoming deuteron at $15 \mathrm{MeV}$ is larger than the yield for protons at $30 \mathrm{MeV}$, the lower current reduces the gains in total neutron number. In addition to that, the lower energy of the beams that would be produced in the ${ }^{9} \mathrm{Be}(\mathrm{d}, \mathrm{nx})$ reaction is expected to reduce the production of very neutron-rich fission products. For these reasons, in the first phase of development of the source we chose to pursue the ${ }^{9} \mathrm{Be}(\mathrm{p}, \mathrm{nx})$ design.

The manuscript is detailed as follows. In sect. 2, we describe the chosen design for the proton-neutron converter along with the studies done with the help of Monte Carlo codes to extract the produced neutron energy spectrum with calculations in FLUKA $[10,11]$ and MCNPX [12]. We also studied heat-removal issues with the finite element code COMSOL Multiphysics ${ }^{\circledR}$.

We will then discuss the experimental techniques used in two characterisation measurements. The first measurement, on a prototype of the converter, was performed at The Svedberg Laboratory (TSL) in Uppsala with an Extended-Range Bonner Sphere Spectrometer (ERBSS) and a liquid scintillator. The second series of measurements was done with Thin-Film Breakdown Counter (TFBC) detectors and activation foils after the converter was deployed at JYFL. In the measurements performed with the liquid scintillator and the TFBCs, the energy of the neutrons was extracted using the time-of-flight (TOF) technique.

The main objective of the first campaign was to obtain a realistic characterisation of the neutron energy spectrum over the entire energy range in a controlled environment, i.e., with a simplified geometry surrounding the detectors and the neutron converter. The second campaign was carried out to obtain a direct measurement of the absolute neutron flux on target and to verify that the shape of the neutron energy spectrum was compatible with the earlier characterisation.

The results of the measurements are presented and compared with Monte Carlo calculations in sect. 3 and further discussed in sect. 4 .

\footnotetext{
${ }^{1}$ COMSOL Multiphysics ${ }^{\circledR}$ v. 5.2. Comsol AB, Stockholm,
} Sweden.

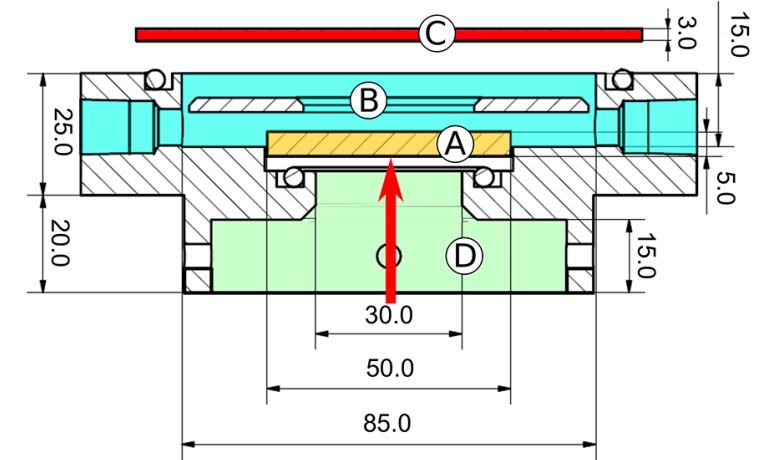

Fig. 1. Exploded view of the proton-neutron converter installed at IGISOL. $30 \mathrm{MeV}$ protons (red arrow) impinge on a $5 \mathrm{~mm}$ thick beryllium disc (A), orange) cooled by water on the backside (B), cyan). A $3 \mathrm{~mm}$ thick aluminium plate (C), red) closes the target assembly and isolates the flowing water from the fission chamber. In this design, the beryllium disc is also used as a window to the vacuum of the beam-line to the cyclotron (D), green). All dimensions are in $\mathrm{mm}$.

\section{Materials and methods}

\subsection{The proton-neutron converter}

Considering the requirements for the total neutron yield and the thermal properties of the converter, we chose a $5 \mathrm{~mm}$ thick beryllium disc cooled by water on the backside. The thickness of the converter is smaller than the range of $30 \mathrm{MeV}$ protons in beryllium. The protons will deposit about $20 \mathrm{MeV}$ in the beryllium and finally stop in the cooling water [7]. This, along with the good thermal properties of beryllium in terms of conductivity and melting point, will allow an effective heat removal, as discussed in more detail in sect. 2.1.2. A schematic diagram of the neutron converter is shown in fig. 1 .

\subsubsection{Neutron energy spectrum}

The neutron energy spectrum was calculated with Monte Carlo simulations, using FLUKA and MCNPX ${ }^{2}$.

The double-differential neutron yield calculated with both codes when $29.6 \mathrm{MeV}$ protons impinge on the converter is shown in fig. 2. The geometry used for the calculations is the same as in the characterisation measurement at TSL, described in detail in sect. 2.2. The neutron yields simulated with the two codes show the same general behaviour, with three main features: a thermal peak (whose main contribution is from neutrons scattering in the experimental hall), an evaporation peak around $1 \mathrm{MeV}$, and a high-energy peak at about $10 \mathrm{MeV}$. MCNPX produces a more intense high-energy peak, while FLUKA predicts a larger contribution from scattered neutrons in the thermal region.

The differences in the simulations further motivate an experimental study to settle the disagreement between the

\footnotetext{
2 The library ENDF70PROT, based on ENDF/B-VII.0 evaluations, was used in MCNPX to model the ${ }^{9} \operatorname{Be}(\mathrm{p}, \mathrm{x})$ reactions.
} 


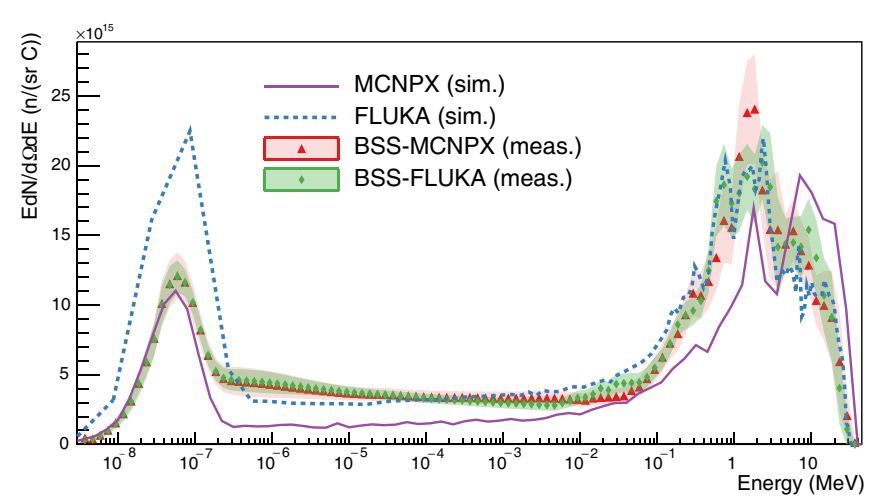

Fig. 2. Results of the double-differential neutron yield measured with the ER-BSS and unfolded using FLUKA- and MCNPX-based guess spectra, compared with simulations of the same geometry calculated with the two codes. The simulations include production and subsequent transport of neutrons in the setup. The yield, plotted in units of lethargy, relates to $29.6 \mathrm{MeV}$ protons impinging on the beryllium converter.

two codes. The actual neutron field was characterised in two experimental campaigns performed before and after the deployment of the converter at JYFL.

\subsubsection{Heat dissipation}

According to the MCC30/15 cyclotron design specifications, the total power of the proton beam can be as high as $3 \mathrm{~kW}(30 \mathrm{MeV} \times 100 \mu \mathrm{A})$. This energy is mostly converted to heat in the materials that the protons interact with. The limited size of the beam spot (a few $\mathrm{mm}$ in diameter) can cause the power to be deposited in a very small volume of the beryllium disc, so that proper cooling of the converter becomes necessary in order to ensure the mechanical stability of the system.

The thickness of the converter was chosen to allow the protons to pass through the beryllium and stop in the cooling water. This avoids hydrogen build-up in the converter, which has proven to be a major problem at similar facilities [13]. At the same time it reduces the requirements for heat removal, since a part of the proton energy is deposited in the cooling water. Production of neutrons in the water is not expected to give a considerable contribution to the total flux; on the other hand, activation in the water will be induced both by the protons directly and by the neutrons produced in the beryllium converter [14].

A COMSOL Multiphysics ${ }^{\circledR}$ simulation confirmed that a flow of water of about $4 \mathrm{l} / \mathrm{min}$ is sufficient to keep the temperature of the beryllium well below $1560 \mathrm{~K}$, i.e. its melting point.

\subsection{Neutron field characterisation at TSL}

The characterisation measurement of the prototype was performed at the PAULA ${ }^{3}$ facility at TSL, Uppsala (Sweden). The energy of the $37 \mathrm{MeV}$ proton beam was reduced

\footnotetext{
${ }^{3}$ Proton fAcility in UppsaLA.
}

to $29.6 \mathrm{MeV}$ by means of a $1 \mathrm{~mm}$ thick aluminium degrader in order to reproduce the maximum energy of the MCC30/15 cyclotron at JYFL. The proton beam, pulsed with a period of $44.25 \mathrm{~ns}$, impinged on the beryllium converter after a multi-stage collimation.

The relative intensity of the proton beam was monitored in real time with a pair of thin scintillators (proton telescope), which detects protons scattered at an angle of approximately $45^{\circ}$ off a stainless steel foil at the end of the beam-pipe, upstream the degrader and the collimators. At this stage, we have no data for an absolute calibration to relate the counts in the proton telescope to the proton current on the converter; we are therefore reporting only relative neutron yields from the TSL campaign.

The converter was embedded in a $15 \times 15 \mathrm{~cm}^{2}$ aluminium frame with a $1 \mathrm{~cm}$ thick cavity on the back filled with water, where the protons stop after passing through the beryllium. This design closely resembles the final design later installed at IGISOL.

\subsubsection{Bonner sphere spectrometer}

An ER-BSS [15] was selected for this experimental campaign for its capability of determining all energy components of the neutron spectrum, from thermal energies up to tens of MeV. The ER-BSS from INFN-LNF [16-18] consists of 14 polyethylene (PE) spheres, labelled with their diameter in inches: $0^{\prime \prime}=$ bare thermal neutron detector, $2^{\prime \prime}, 2.5^{\prime \prime}, 3^{\prime \prime}, 3.5^{\prime \prime}, 4^{\prime \prime}, 4.5^{\prime \prime}, 5^{\prime \prime}, 7^{\prime \prime}, 8^{\prime \prime}, 10^{\prime \prime}$, plus three highenergy spheres, called ERS-1, ERS-2 (7" external diameter, with an internal $4^{\prime \prime} \mathrm{PE}$ sphere surrounded by $1.27 \mathrm{~cm}$ of lead or copper, respectively) and ERS-3 (12" external diameter, with an internal 3.15" PE sphere surrounded by $1 \mathrm{~cm}$ of lead). The central thermal neutron detector is a cylindrical $4 \times 4 \mathrm{~mm}{ }^{6} \mathrm{LiI}(\mathrm{Eu})$ scintillator, whose pulse height distribution is used to discriminate thermal neutrons from the photon contribution. The ER-BSS response matrix is known with an overall uncertainty of approximately $3 \%[19]$. The calibration factor of this ER-BSS is verified regularly with an $\mathrm{NPL}^{4}$-calibrated ${ }^{241} \mathrm{Am}-\mathrm{Be}$ source and its maintenance is guaranteed through a routine quality assurance program.

A known limitation of such a system is the poor energy resolution above $5 \mathrm{MeV}$ [20], due to the similarities in the spheres' response functions. As a consequence, Bonner spheres data must be complemented with a certain amount of a priori information in order to obtain the resulting neutron spectrum (unfolding). This can be done by providing a guess spectrum, typically from Monte Carlo simulations. A statistical package, built into the FRUIT deconvolution code $[16,21,22]$, propagates uncertainties of input quantities through the unfolding process, thus obtaining distribution probabilities and uncertainties for the neutron spectrum on a bin-by-bin basis.

\footnotetext{
${ }^{4}$ National Physical Laboratory, UK (www.npl.co.uk).
} 


\subsubsection{Liquid scintillator}

To complement the ER-BSS, a TOF system was used to obtain a high-resolution measurement in the energy range above $6 \mathrm{MeV}$.

The TOF setup consisted of a 3.31 BC-501 liquid scintillator from the NORDBALL array [23]. The scintillator was read out by a digital acquisition system based on an SP Devices ADQ412 high-speed digitizer. The TOF was extracted as the time difference between the signal coming from the scintillator, which acted as a trigger, and a stop signal that was phase-locked to the proton pulses, given by the radio frequency (RF) of the cyclotron. Both signals were recorded as digital pulses by the ADQ412 on an event-by-event basis.

Multiple $\gamma$ sources were used to extract the pulseheight resolution and to calibrate the energy of the liquid scintillator. Details on the characterisation of the detector are discussed in ref. [14].

\subsection{Characterisation of the neutron field at JYFL}

The converter was first installed at IGISOL in April 2014 and the neutron flux was measured with TFBCs. An activation measurement was performed in parallel and has been reported elsewhere $[6,7]$.

\subsubsection{Thin-Film Breakdown Counters}

TFBCs are selectively sensitive to highly ionizing particles. The detectors are built as a sandwich of two electrodes, to which a voltage is applied, interleaved with a dielectric layer. In a TFBC, an electrical breakdown is detected, which is produced when a highly ionizing particle creates a conductive channel through the insulator. If a fission target is placed on top of one of the electrodes, neutrons can indirectly be detected through the fission products [24-26]. Due to the fast time response, below approximately $1 \mathrm{~ns}$, TFBCs can be used for TOF measurements.

The count rate of the TFBC depends on the detector efficiency and the fission rate in the target. The latter depends on the properties of the target applied to the TFBC detector (the material used and its thickness), as well as on the neutron flux and energy spectrum [24-26].

\section{Results}

\subsection{Neutron field characterisation at TSL}

\subsubsection{ER-BSS}

The fourteen spheres described in the previous sect. were exposed in sequence at the point of test $(180 \mathrm{~cm}$ from the
Table 1. Energy-integrated quantities for the ER-BSS spectra unfolded with FRUIT-SGM using FLUKA- and MCNPXbased guess spectra.

\begin{tabular}{crr}
\hline & \multicolumn{2}{c}{ Unfolding } \\
& FLUKA-based & MCNPX-based \\
& $\left(10^{4} \mathrm{n} / \mathrm{sr} / \mathrm{MU}\right)$ & $\left(10^{4} \mathrm{n} / \mathrm{sr} / \mathrm{MU}\right)$ \\
\hline$E<0.4 \mathrm{eV}$ & $1.87 \pm 0.48$ & $1.89 \pm 0.84$ \\
$0.4 \mathrm{eV}-10 \mathrm{keV}$ & $2.44 \pm 0.05$ & $2.51 \pm 0.06$ \\
$10 \mathrm{keV}-1 \mathrm{MeV}$ & $2.63 \pm 0.09$ & $2.42 \pm 0.10$ \\
$1 \mathrm{MeV}-6 \mathrm{MeV}$ & $1.98 \pm 0.12$ & $2.12 \pm 0.15$ \\
$E>6 \mathrm{MeV}$ & $1.33 \pm 0.11$ & $1.28 \pm 0.12$ \\
\hline Total Yield & $10.24 \pm 0.32$ & $10.21 \pm 0.32$ \\
\hline
\end{tabular}

proton-neutron converter at a $10^{\circ}$ angle from the beamaxis in the forward direction). Their readings were normalized to the counts of the proton telescope, thus obtaining the BSS counts per Monitor Unit $(\mathrm{MU})^{5}$. The normalized ER-BSS counts were unfolded using the FRUIT code with the function FRUIT-SGM (Special Gradient Method, with guess spectrum). Realistic guess spectra were derived from FLUKA and MCNPX simulations.

The unfolded spectra obtained with FRUIT using the FLUKA-based or MCNPX-based guess spectrum are compatible within the provided uncertainties (see fig. 2). These uncertainties are smaller than $15 \%$ for energies below $5 \mathrm{MeV}$, between $15 \%$ and $30 \%$ in the 5 to $20 \mathrm{MeV}$ range, and approximately $30 \%$ above $20 \mathrm{MeV}$. Obtaining compatible unfolded spectra with different guess spectra is an indication of the robustness of the code.

Table 1 reports the spectrum-integrated total neutron yield and neutron yield fractions under specific energy intervals. Again, there are no significant differences when unfolding the data with a FLUKA- or MCNPX-based guess spectrum.

In fig. 2, the integral of the ER-BSS measurement above $0.5 \mathrm{eV}$ was rescaled to match the integral of the FLUKA simulation in the same energy range ${ }^{6}$. In this range, the shape of the simulation is in very good agreement with the measurement result. At thermal energies, the measurement is better described by MCNPX, while FLUKA overestimates the contribution from scattered neutrons.

\subsubsection{TOF with a liquid scintillator}

Measurements with the liquid scintillator have been made at three different distances from the converter, at a $10^{\circ}$ angle: $1.2 \mathrm{~m}, 2.1 \mathrm{~m}$ and $4.9 \mathrm{~m}$. This choice was made to

\footnotetext{
$51 \mathrm{MU}$ corresponds to 1 count in the proton telescope monitor.

6 The ratio of the integrals, i.e. the factor used to rescale the ER-BSS spectrum is $1.4 \times 10^{12}$.
} 
reach a compromise between the energy resolution and the detrimental effect of the wrap-around ${ }^{7}$, both increasing with distance. In the offline analysis, a cut on the pulse-height was implemented. It was increased with the measurement distance to avoid the disturbance due to the wrap-around. To take advantage of the best settings for the 3 distances (i.e., highest possible energy resolution and absence of wrap-around) the results from the different measurements have then been combined over the energy range between 6 and $30 \mathrm{MeV}$, by scaling for the change in solid angle covered by the detector. Runs with shadow cones were used to subtract the contribution of scattered neutrons.

The liquid scintillator allowed discrimination between neutrons and photons on the basis of the pulse shape. The Pulse Shape Discrimination (PSD) was performed offline on the digitised pulses using the charge integration method. Pulses corresponding to neutrons were selected based on both a PSD parameter and the pulse height, the procedure is described in more detail in ref. [14].

Absolute timing in the TOF spectra was obtained from the $\gamma$ flash generated by the protons when they hit elements along the beam-line (i.e., the aluminium degrader, the different collimators and the converter itself). From the duration of the $\gamma$ pulse, the time resolution of the system could be evaluated to be approximately $1.5 \mathrm{~ns}$ FWHM, that translates to an energy resolution of $0.8 \mathrm{MeV}$ at $30 \mathrm{MeV}$ [14].

A response function of the liquid scintillator to neutrons of different energies has been extracted for each threshold level, using the pulse height information from the digital DAQ system and a Monte Carlo calculation performed in Geant4, using the CHIPS-TPT library $[14,27,28]$ and the light output for protons, deutrons and alpha particles from refs. [29,30]. The CHIPS-TPT library was chosen for its treatment of the ${ }^{12} \mathrm{C}(\mathrm{n}, \mathrm{n}) 3 \alpha$ reaction, after a dedicated study showed that it was able to reproduce measured efficiency data better than any of the other main libraries available in Geant4. This study is discussed in detail in ref. [14] and the efficiency curves are shown in fig. 3 .

In fig. 4, the double-differential yield measured with the TOF technique is compared to the MCNPX and FLUKA calculations above $5 \mathrm{MeV}$. Included are also the ER-BSS spectrum over the same energy range and the result of a similar TOF measurement by Johnsen [31], in which $30 \mathrm{MeV}$ protons from the cyclotron at the University of California, Davis, impinged on a $1.27 \mathrm{~cm}$ thick beryllium converter.

Based on a $\chi^{2}$-test, we found that the TOF measurement with the liquid scintillator best agrees with the MCNPX simulation. We therefore chose to normalize our data

\footnotetext{
7 Wrap-around or frame-overlap is the superposition of flight times of the neutrons due to the repetition rate of a pulsed neutron source. This causes ambiguities in the determination of the energy, since a slow neutron may be mistaken for a fast neutron from a subsequent pulse.
}

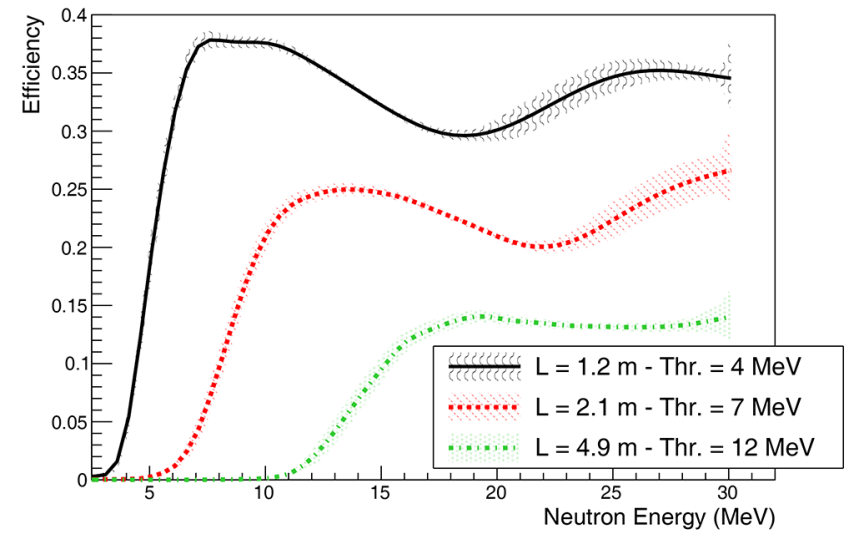

Fig. 3. Scintillator detector efficiency curves for the thresholds used in the TOF measurement at a distance of 1.2 (solid black), 2.1 (dashed red) and $4.9 \mathrm{~m}$ dash-dotted green). The uncertainties are indicated with a shade of the same colour.

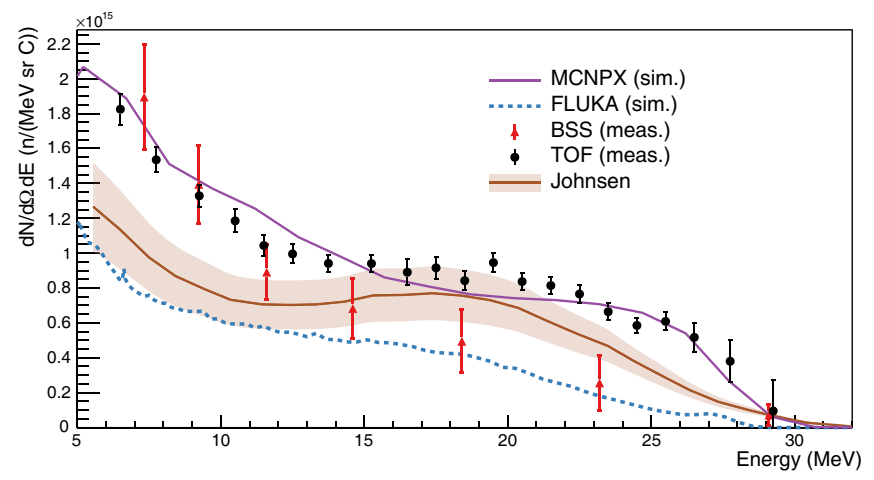

Fig. 4. Neutron energy spectra from the TSL measurement with the TOF and the ER-BSS (using the MCNPX guess spectrum). The experimental results are compared to Monte Carlo simulations in FLUKA and MCNPX, as well as to Be(p, nx) data measured by Johnsen [31]. The normalization of the histograms is discussed in the text.

to the integral of the MCNPX simulation over the energy range where the spectra overlap ${ }^{8}$.

\subsection{Characterisation of the converter deployed at JYFL}

After the installation of the converter at IGISOL, a validation measurement was performed with TFBCs using the TOF technique.

A ${ }^{252} \mathrm{Cf}$ source was used to measure the efficiency of the detectors. The efficiency is defined as the product of the sensitive area of the TFBC and the intrinsic efficiency for detection of fission fragments. This value was found to be $(0.176 \pm 0.009) \mathrm{cm}^{2}$.

8 The ratio of the integrals is $2.1 \times 10^{12}$. A realistic estimate of the uncertainty on the normalization factor is of the order of $20 \%$; this is dominated by the uncertainty on the absolute efficiency of the liquid scintillator, only determined with Monte Carlo calculations [14]. 


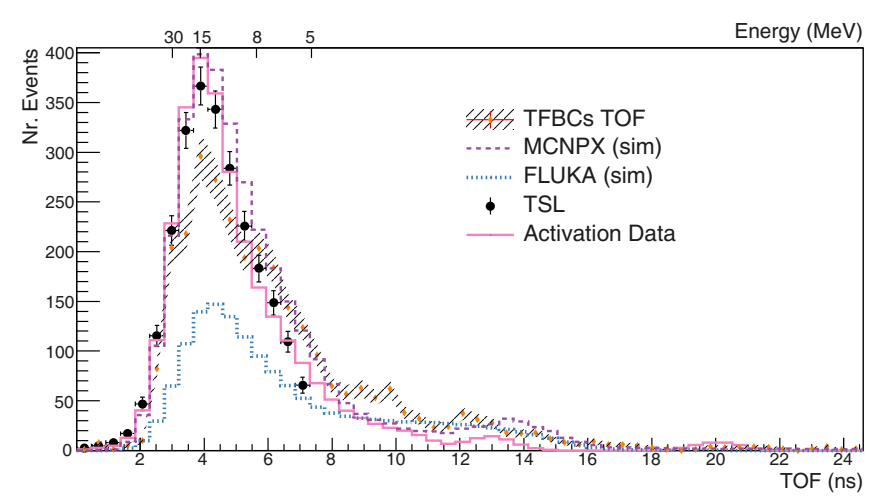

Fig. 5. Results of the measurement of the neutron TOF spectrum inside the fission chamber at IGISOL, JYFL. All data sets are in absolute units of counts in the TFBCs; the TSL data use the same normalization as in fig. 4. An approximate neutron energy scale is shown on the top axis.

The TFBC was positioned at a distance of $25.5 \mathrm{~cm}$ from the backside of the converter in the direction of the beam; the centre of the TFBC was located at $1.2 \mathrm{~cm}$ and $3.2 \mathrm{~cm}$ to the right and top, respectively, from the centre of the beryllium disc.

An $80 \mu \mathrm{g} / \mathrm{cm}^{2}$ isotopically pure ${ }^{238} \mathrm{U}$ foil was used as the TFBC fission target. Irradiation was carried out for $907 \mathrm{~s}$ at a proton beam current of $2.1 \mu \mathrm{A}$.

Because of the short flight-path of the neutrons between production and detection, we chose to compare the result of this measurement with other neutron spectra in terms of TFBC counts in the TOF-frame. This reduces the uncertainty that would be introduced if the spectrum measured with the TFBC was to be corrected for the energydependent response function.

The neutron energy spectra calculated with Monte Carlo codes or measured with other experimental techniques were then convoluted with the TFBC response function $(\varepsilon(E))$. Including the information on the integrated proton current $(Q)$, the incident fluence at the irradiation position $(\Phi(E))$ was converted to the number of events that would be detected by the TFBC $\left(T F B C_{\text {eq }}\right)$ if it was exposed to such a neutron field

$$
T F B C_{\mathrm{eq}}(E)=Q \cdot \Phi(E) \cdot \varepsilon(E) .
$$

The result of the MCNPX calculation was provided as a spectral neutron fluence in TOF, so that it could be directly compared to experimental data, after including the corrections mentioned above. The other spectra, instead, have been converted from the energy- to the TOF-frame in the position where the TFBC was placed. A Gaussian spread was added in order to account for the time resolution of the TOF system (estimated to be $1.7 \mathrm{~ns}$ FWHM).

Figure 5 shows the result of the TOF measurement with the TFBC, compared to the activation measurement and the Monte Carlo simulations of the same experimental setup, in the TOF-frame. The spectrum from the TSL characterisation is also shown.

\section{Discussion}

In this work, we aim at a characterisation of the neutron field from the proton-neutron converter designed for the IGISOL facility at JYFL.

We compared the measurements of the neutron fields performed at the TSL and JYFL facilities using different experimental techniques, despite the different conditions for the measurements in terms of background, neutron scattering and distance from the source. The most obvious effect of these differences, in fact, would be seen in the lower energy range, a portion of the spectrum that is of limited interest for application of the source in n-induced fission, especially in the case of fertile nuclei like ${ }^{238} \mathrm{U}$ and ${ }^{232} \mathrm{Th}$, which are commonly used targets.

Results from the campaign at TSL are only on a relative scale; these, however, give very valuable information in terms of the shape of the neutron energy spectrum, thanks to the superior energy resolution of the TOF system, with respect to the one used at JYFL. The absolute neutron yield was instead obtained from the campaign at JYFL, from where a total neutron flux between 2 and $5 \times 10^{12} \mathrm{n} / \mathrm{sr} / \mathrm{s}$ at an incoming proton current of $100 \mu \mathrm{A}$ can be estimated. Of these, between 2 and $3 \times 10^{12} \mathrm{n} / \mathrm{sr} / \mathrm{s}$ are fast neutrons $\left(E_{\mathrm{n}}>1 \mathrm{MeV}\right)[14]$.

The shape of the TOF measurement at TSL is best reproduced by the MCNPX simulation. It is also in reasonable agreement with the measurement by Johnsen, performed on a similar setup. The difference in shape observed above $25 \mathrm{MeV}$ can be attributed to the different energy resolution of the two detectors (approximately $3 \mathrm{MeV}$ at $30 \mathrm{MeV}$ for Johnsen's [31] vs. $0.8 \mathrm{MeV}$ of our system). It should be noted that, in fig. 4, the energy resolution on Johnsen's data is not included in the error bars, that only represent the uncertainty on the differential neutron yield attributed by Johnsen to the efficiency calibration [31].

The ER-BSS result agrees better with the FLUKA simulation at energies above $0.5 \mathrm{eV}$. The thermal peak, caused by neutrons scattering in the experimental room, is instead better described by MCNPX.

Despite the numerous investigations and tests performed to reconcile the two Monte Carlo codes, we found no good explanation for the differences observed in the high-energy region of the neutron spectrum. With respect to the experimental techniques, we rely more on the TOF results in the fast energy range. Attempts to extract detailed information on the spectrum shape above $6 \mathrm{MeV}$ are not very meaningful for ER-BSS, because this region represents a small fraction of the total fluence (about 13.0\%) in an energy range where the ER-BSS shows a sub-optimal energy resolution due to similar response functions of the Bonner Spheres. ER-BSS, on the other hand, is the only technique which is able to cover the whole spectrum.

The result of the TFBC measurement at IGISOL agrees better in absolute scale with the MCNPX simulation. The MCNPX-simulated neutron flux also well reproduces the result of the activation measurement $[6,7]$.

We therefore conclude that the MCNPX code describes sufficiently well the high-energy region of the neu- 
tron energy spectrum from the $\mathrm{Be}(\mathrm{p}, \mathrm{nx})$ reaction, while FLUKA underestimates it.

\section{Summary}

$\mathrm{A} \operatorname{Be}(\mathrm{p}, \mathrm{nx})$ neutron source has been designed for neutroninduced fission yields studies. The source will use highcurrent $30 \mathrm{MeV}$ protons from the MCC30/15 cyclotron installed at the IGISOL facility at JYFL. The neutron yield has been calculated with the Monte Carlo codes FLUKA and MCNPX.

A characterisation measurement was performed at the TSL, Uppsala (Sweden), where a mock-up of the protonneutron converter was irradiated with $29.6 \mathrm{MeV}$ protons. The energy spectrum was measured with two techniques: an ER-BSS to measure from thermal energies up to $30 \mathrm{MeV}$; and a TOF system, to focus on the high-energy part of the spectrum (above $6 \mathrm{MeV}$ ).

As far as the ER-BSS is concerned, separate unfolding procedures have been used starting from FLUKA- and MCNPX-based guess spectra in the FRUIT-SGM code. The final spectrum does not depend on the guess spectrum adopted and well reproduces the FLUKA simulation above $0.5 \mathrm{eV}$.

The shape of the neutron spectrum in the fast energy range, as measured using the TOF technique, is well described by the MCNPX simulation, i.e. the spectrum is harder than suggested by FLUKA.

The neutron field produced by the final design of the neutron source was also measured at JYFL, in a position very close to where the target for neutron-induced fission studies will be placed. The results from this campaign, using the TFBC detectors and the activation foils, are in reasonable agreement with the MCNPX simulation and confirmed that the energy spectrum is compatible with the one measured in the first characterisation at TSL.

Overall, we conclude that three different measurements (TOF with liquid scintillator, TOF with TFBCs and activation foils), as well as the earlier measurement by Johnsen, support the harder neutron-energy spectrum suggested by MCNPX.

According to the measured fission rates with the TF$\mathrm{BCs}$, the design goal, in terms of both total and fast neutron flux, is fulfilled, which will allow neutron-induced fission studies at IGISOL-JYFLTRAP.

This work was supported by the European Commission within the Seventh Framework Programme through Fission-2010ERINDA (project no. 269499) and Fission-2013-CHANDA (project no. 605203), by the NESCOFI@BTF project (INFN - CSN V, Italy), by the Swedish Radiation Safety Authority (SSM), and by the Swedish Nuclear Fuel and Waste Management Co. (SKB).

Open Access This is an open access article distributed under the terms of the Creative Commons Attribution License (http://creativecommons.org/licenses/by/4.0), which permits unrestricted use, distribution, and reproduction in any medium, provided the original work is properly cited.

\section{References}

1. A. Solders, D. Gorelov, A. Jokinen et al., Nucl. Data Sheets 119, 338 (2014).

2. P. Dimitriou, F.-J. Hambsch, S. Pomp, Fission Yields: current status $\&$ perspective. Summary report of an IAEA Technical Meeting, Technical Report INDC(NDS)-0713, IAEA, 2016

3. H. Penttilä, V.-V. Elomaa, T. Eronen et al., Eur. Phys. J. A 48, 1 (2012).

4. H. Penttilä, D. Gorelov, V.-V. Elomaa et al., Eur. Phys. J. A 52, 1 (2016).

5. J. Äystö, T. Eronen, A. Jokinen et al., IGISOL: Three Decades of Research Using IGISOL Technique at the University of Jyväskylä (Springer Science \& Business Media, 2014).

6. D. Gorelov, Nuclear Fission Studies With the IGISOL Method and JYFLTRAP, $\mathrm{PhD}$ Thesis, University of Jyväskylä, (2015).

7. D. Gorelov, H. Penttilä, A. Al-Adili et al., Nucl. Instrum. Methods Phys. Res. B 376, 46 (2016).

8. M. Lantz, D. Gorelov, A. Jokinen et al., Design of a high intensity neutron source for neutron-induced fission yield studies, in Annex: Individual Reports to Compendium of Neutron Beam Facilities for High Precision Nuclear Data Measurements, (IAEA-TECDOC-1743, 2014) p. 234.

9. I.D. Moore, T. Eronen, D. Gorelov et al., Nucl. Instrum. Methods Phys. Res. B 317, 208 (2013).

10. T.T. Böhlen, F. Cerutti, M.P.W. Chin et al., Nucl. Data Sheets 120, 211 (2014).

11. A. Ferrari, P.R. Sala, A. Fasso, J. Ranft, FLUKA: A multiparticle transport code (Program version 2005), Technical report, 2005.

12. L.S. Waters, G.W. McKinney, J.W. Durkee et al., The MCNPX Monte Carlo radiation transport code, in Hadronic Shower Simulation Workshop, Vol. 896, (AIP Publishing, 2007) pp. 81-90.

13. T. Rinckel, D.V. Baxter, J. Doskow et al., Phys. Procedia 26, 161 (2012).

14. A. Mattera, Characterization of a neutron source for fission yields studies, Licentiate Thesis, Uppsala, (2014), available from: http://urn.kb.se/resolve?urn= urn:nbn:se:uu:diva-238044.

15. B. Wiegel, A.V. Alevra, Nucl. Instrum. Methods Phys. Res. A 476, 36 (2002).

16. S. Agosteo, R. Bedogni, M. Caresana et al., Nucl. Instrum. Methods Phys. Res. A 694, 55 (2012).

17. K. Amgarou, R. Bedogni, C. Domingo et al., Nucl. Instrum. Methods Phys. Res. A 654, 399 (2011).

18. R. Bedogni, K. Amgarou, C. Domingo et al., Nucl. Instrum. Methods Phys. Res. A 681, 110 (2012).

19. R. Bedogni, C. Domingo, A. Esposito et al., Nucl. Instrum. Methods Phys. Res. A 620, 391 (2010).

20. M. Reginatto, Nucl. Instrum. Methods Phys. Res. A 480, 690 (2002).

21. R. Bedogni, C. Domingo, A. Esposito, F. Fernández, Nucl. Instrum. Methods Phys. Res. A 580, 1301 (2007).

22. R. Bedogni, M. Pelliccioni, A. Esposito, Nucl. Instrum. Methods Phys. Res. A 615, 78 (2010).

23. S.E. Arnell, H.A. Roth, Ö. Skeppstedt et al., Nucl. Instrum. Methods Phys. Res. A 300, 303 (1991). 
24. L. Tommasino, N. Klein, P. Solomon, J. Appl. Phys. 46, 1484 (1975).

25. V.P. Eismont, A.V. Prokofiev, A.N. Smirnov, Radiat. Meas. 25, 151 (1995).

26. A.N. Smirnov, A. Pietropaolo, A.V. Prokofiev et al., Nucl. Instrum. Methods Phys. Res. A 687, 14 (2012).

27. M. Kosov, D. Savin, J. Phys. Conf. Ser. 608, 012050 (2015).
28. M.V. Kossov, Manual for the CHIPS event generator, KEK, internal report 17-2000 (2001).

29. V. Verbinski, W. Burrus, T. Love et al., Nucl. Instrum. Methods 65, 8 (1968).

30. N. Nakao, T. Nakamura, M. Baba et al., Nucl. Instrum. Methods Phys. Res. A 362, 454 (1995).

31. S.W. Johnsen, Phys. Med. Biol. 23, 499 (1978). 